\title{
Association of polybrominated diphenyl ether (PBDE) levels with biomarkers of placental development and disease during mid-gestation
}

Julia R. Varshavsky ${ }^{1 *+}$ (D) Joshua F. Robinson ${ }^{1,2+}$, Yan Zhou ${ }^{2}$, Kenisha A. Puckett ${ }^{2}$, Elaine Kwan², Sirirak Buarpung ${ }^{2}$, Rayyan Aburajab², Stephanie L. Gaw ${ }^{2,3}$, Saunak Sen ${ }^{4}$, Sabrina Crispo Smith ${ }^{5}$, Julie Frankenfield ${ }^{5}$, June-Soo Park, Susan J. Fisher ${ }^{2}$ and Tracey J. Woodruff ${ }^{1}$

\begin{abstract}
Background: Polybrominated diphenyl ether (PBDE) exposures have been associated with adverse pregnancy outcomes. A hypothesized mechanism is via alterations in placental development and function. However, we lack biomarkers that can be used as early indicators of maternal/fetal response to PBDE exposures and/or perturbations in placental development or function.

Methods: To evaluate the relationship between PBDE levels and placental biomarkers during mid-gestation of human pregnancy $(n=62)$, we immunolocalized three molecules that play key roles in cytotrophoblast (CTB) differentiation and interstitial/endovascular uterine invasion-integrin alpha-1 (ITGA1), vascular endothelial-cadherin (CDH5), and metalloproteinase-1 (MMP1)-and assessed three morphological parameters as potential indicators of pathological alterations using H\&E-stained tissues-leukocyte infiltration, fibrinoid deposition, and CTB endovascular invasion. We evaluated associations between placental PBDE levels and of biomarkers of placental development and disease using censored Kendall's tau correlation and linear regression methods.
\end{abstract}

Results: PBDEs were detected in all placental samples. We observed substantial variation in antigen expression and morphological endpoints across placental regions. We observed an association between PBDE concentrations and immunoreactivity of endovascular CTB staining with anti-ITGA1 (inverse) or interstitial CTBs staining with anti-CDH5 (positive)

\footnotetext{
* Correspondence: julia.varshavsky@ucsf.edu

${ }^{\dagger}$ Julia R. Varshavsky and Joshua F. Robinson joint first authors

'Program on Reproductive Health and the Environment, UCSF Department of Obstetrics, Gynecology \& Reproductive Sciences, University of California, Mailstop 0132, 550 16th Street, 7th Floor, San Francisco, CA 94143, USA

Full list of author information is available at the end of the article
}

C C The Author(s). 2020 Open Access This article is licensed under a Creative Commons Attribution 4.0 International License, which permits use, sharing, adaptation, distribution and reproduction in any medium or format, as long as you give appropriate credit to the original author(s) and the source, provide a link to the Creative Commons licence, and indicate if changes were made. The images or other third party material in this article are included in the article's Creative Commons licence, unless indicated otherwise in a credit line to the material. If material is not included in the article's Creative Commons licence and your intended use is not permitted by statutory regulation or exceeds the permitted use, you will need to obtain permission directly from the copyright holder. To view a copy of this licence, visit http://creativecommons.org/licenses/by/4.0/. The Creative Commons Public Domain Dedication waiver (http://creativecommons.org/publicdomain/zero/1.0/) applies to the data made available in this article, unless otherwise stated in a credit line to the data. 
(Continued from previous page)

Conclusions: We found several molecular markers that may be sensitive placental indicators of PBDE exposure. Further, this indicates that placental biomarkers of development and disease could be useful barometers of exposure to PBDEs, a paradigm that could be extended to other environmental chemicals and placental stagespecific antigens.

Keywords: Flame retardants, Biomonitoring, Developmental/reproductive health effects, Endocrine disruption, Preeclampsia, Pregnancy complications, Maternal health, Birth outcomes, Cytotrophoblast differentiation

\section{Background}

Pregnancy complications, such as preterm birth, gestational diabetes, fetal growth restriction, hypertension, and preeclampsia, are significantly linked with adverse maternal and fetal health outcomes [1], and environmental exposures are important risk factors [2-4]. Environmental chemicals can exert their influence on pregnancy through multiple mechanisms, including alterations in placental development and function.

The placenta plays an essential role in development, supporting embryonic/fetal growth and maternal adaptations to the changing physiology of pregnancy $[5,6]$. The human placenta consists of tree-like chorionic villi originating from fetal cells that float in maternal blood (floating villi, FV; Fig. 1). Mononuclear cytotrophoblast (CTB) progenitors (precursor cells) fuse to form multinucleated syncytiotrophoblasts (STBs) that cover the villus surface where they regulate the exchange of nutrients, waste, and gases. A subset of chorionic villi called anchoring villi (AV) attach the placenta to the uterus. CTBs migrate through the AV and form cell columns that are the source of the extravillous (invading the uterus) subpopulation which invades the uterus and maternal vasculature. Vascular CTB remodeling of uterine arteries redirects blood flow to the placenta, maximizing nutrient uptake for embryonic/fetal growth and development over the course of gestation [7]. During normal pregnancy, modulation of numerous molecules

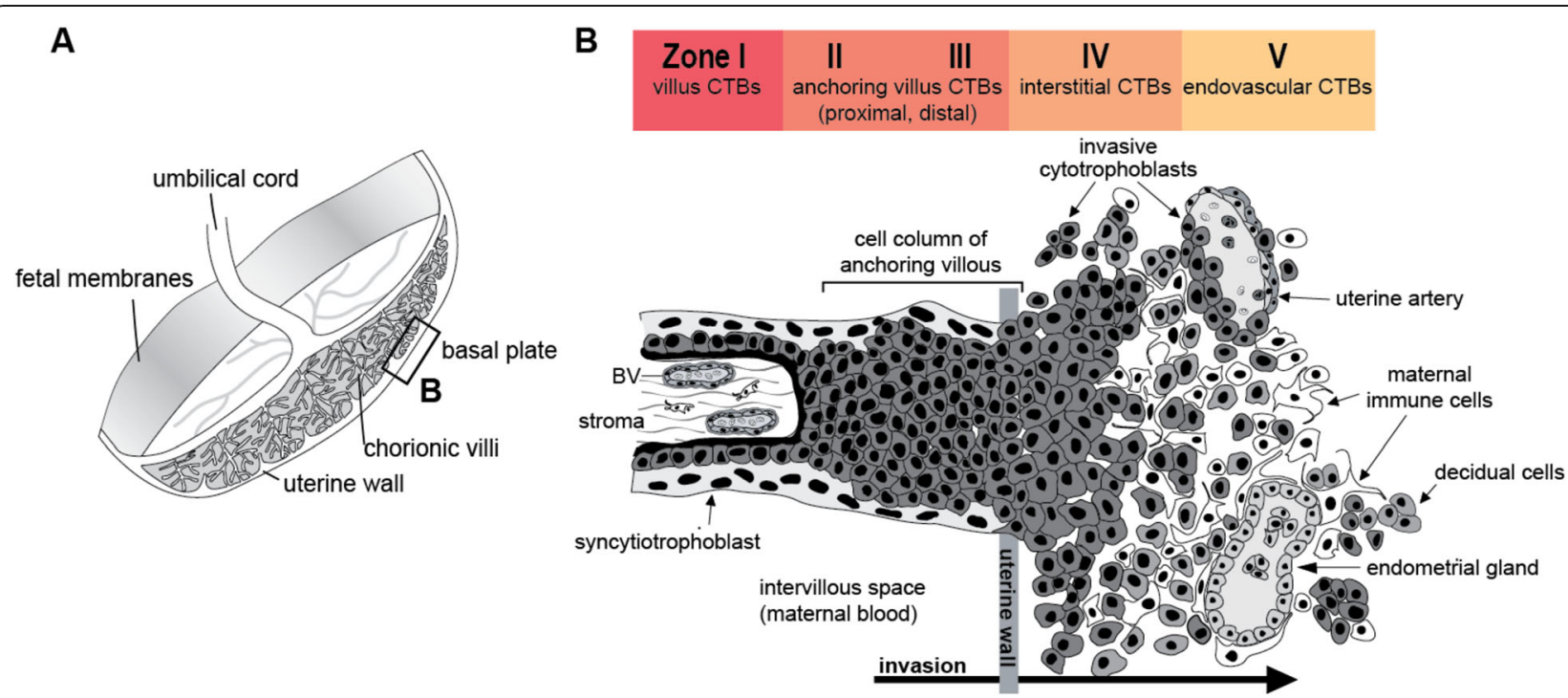

Fig. 1 Human placental villous cytotrophoblast (CTB) differentiation at the maternal-fetal interface. a Anatomy of the human placenta. Chorionic villi are the functional units. The histology of the boxed area is shown in the panel to the right. $\mathbf{b}$ Depiction of the maternal-fetal interface at the cellular level. The mononuclear cytotrophoblasts (CTB) of the (early gestation) chorionic villi fuse to become multinuclear syncytiotrophoblasts (STBs), which form the surface of the placenta. Floating villi are perfused by maternal blood. Anchoring villi give rise to invasive interstitial CTBs (CTBi) that emigrate from the chorionic villi via cell columns that attach the placenta to the maternal unit and infiltrate the uterine wall. Maternal cells in this region include the decidua, remodeled uterine blood vessels, which are lined by cytotrophoblasts (CTBe), and immune cells. During vascular invasion, the cells breach both veins and arteries, but they have more extensive interactions with the arterial portion of the uterine vasculature. Here, they replace the endothelial lining and intercalate within the smooth muscle walls of the spiral arteries, producing hybrid vessels that are composed of both embryonic/fetal and maternal cells. Vascular invasion connects the uterine circulation to the intervillous space where maternal blood perfuses the chorionic villi. Immunoreactivity of molecular biomarkers was evaluated in five zones (I-V) corresponding to different stages of CTB differentiation: I) CTB progenitors in floating villi (FV); II) CTBs of the proximal (AVP) and III) distal (AVd) anchoring villi; IV) invading interstitial CTBs (CTBi); and V) endovascular CTBs (CTBe) that remodel the uterine vasculature. Image modified from Maltepe and Fisher, 2015; Damsky et al., 1992 (Damsky et al., 1992; Maltepe and Fisher, 2015) 
characterized by migratory and invasive properties facilitate CTB differentiation along this pathway: extracellular matrix (ECM)-degrading metalloproteinases (e.g., MMPs), cell-ECM (e.g., integrins), and cell-cell adhesion molecules (e.g., cadherins). The net effect is a unique epithelial (non-invasive CTBs of the anchoring villi cell column) to vascular (invasive CTBs that resemble endothelial cells and colonize maternal blood vessels) transformation [8] critical for placental development and function. For example, CTBs upregulate the expression of alpha 5/beta 1 and alpha 1/beta 1 integrin pairs (ITGA5/B1, A1/B1) while down regulating integrin alpha 6/beta 4 (ITGA6/B4) [9]. Furthermore, as CTBs become invasive and enter cell columns, they down regulate $\mathrm{CDH} 1$ and up regulate $\mathrm{CDH} 5$ (VE-cadherin) [7, 9-11]. Placental development (placentation) is especially critical during mid-gestation, an important time of fetal organogenesis and extreme changes to maternal physiology [4].

Disruption of the balance of adhesion receptor molecules and/or matrix-degrading proteins is associated with pregnancy complications (e.g., preeclampsia, fetal growth restriction, and preterm birth) that can be due to alterations in CTB differentiation which lead to incomplete vascularization [12-16]. For example, in severe preeclampsia, CTB remodeling of maternal vasculature is incomplete, which is thought to contribute to shallow placentation and reduced arterial invasion that are the hallmarks of this syndrome [15]. While specialized subpopulations of immune cells co-occupy the pregnant uterus with placental CTBs, aiding in vascular remodeling [17], excessive white blood cell (leukocyte) infiltration can indicate infection, a major risk factor for preterm birth [18]. Excessive perivillous (fetal side) fibrinoid deposition is also associated with pregnancy complications that either directly or indirectly involve abnormal placentation [19].

Diverse environmental exposures-including heavy metals (i.e., lead and cadmium [20, 21]) and common classes of environmental chemicals (ECs), such as polybrominated diphenyl ethers (PBDEs), per- and polyfluorinated alkyl substances (PFAS), and phthalates-are suspected to contribute to maternal health and pregnancy complications by interfering with placental development and function [22]. However, we know very little about the precise mechanisms by which ECs exert their toxic effects. Despite ongoing efforts to restrict their use as flame retardants, PBDEs remain a global public health concern due to their environmental and biological persistence [23]. Human pregnancy is a period of high sensitivity and susceptibility; meanwhile, PBDEs are commonly identified in placental/fetal tissues [24, 25], and exposures have been associated with maternal health complications [4], adverse birth outcomes [26], and postnatal neurodevelopmental deficits (i.e., lowered IQ [27];). North Americans typically have higher body burden levels due to historically strict flammability standards in the United States (implemented in the 1970s), with some of the highest concentrations ever reported among pregnant women in the State of California [28].

In humans, the relationship between PBDE exposures and pregnancy complications (e.g., preeclampsia) remains undefined. Eslami et al. (2016) found a significant association between total PBDEs and preeclampsia among first mothers in Iran, while no association was detected in a prospective U.S. cohort $[29,30]$. In mice and/or human cell lines, PBDEs alter hormone production [31], elicit inflammation [32-34], and promote oxidative stress [32, 35-39]. Data from in vitro studies supports the hypothesis that PBDEs disrupt trophoblast function and placentation. In human primary CTBs (2nd trimester), BDE-47 impairs CTB invasion and migration as well as causes significant changes on the transcriptomic and methylomic level in pathways which regulate placental development [40]. Additional studies using an extravillous trophoblast cell line (HTR-8/SVneo) have shown BDE-47 to be linked with oxidative stress and pro-inflammatory mediators, potential contributors to impaired CTB invasion, as well as placental dysfunction and disease [32, 41]. Together, these studies indicate a potential role for PBDEs in the pathophysiology of adverse pregnancy outcomes via effects on the placenta and highlight functional and molecular perturbations to human placental development that can be evaluated in relation to PBDE exposures.

In this study, we assessed a time period that is a vulnerable window of CTB invasion when remodeling occurs. Perturbations to this developmental process contribute to preeclampsia and other complications usually clinically identified later in pregnancy or at term. Specifically, we examined the relationship between midgestational PBDE concentrations and biomarkers of placental development and disease, focusing on molecular and morphological features that: 1) correlate with normal placentation and pregnancy outcomes; 2) are adversely affected in pregnancy complications; and/or 3) are differentially expressed following exposure of primary human CTBs to PBDEs in vitro [40]. The molecules were stage-specific CTB antigens that correlate with differentiation and invasion: integrin alpha 1 (ITGA1), VE-cadherin (CDH5), and metalloproteinase 1 (MMP1). The morphological features evaluated were leukocyte infiltration, fibrinoid deposition, and CTB arterial vascular remodeling. This study will advance new methods and findings that will contribute to better understanding the relationship between biomarkers of chemical exposure and potential biomarkers of placental development and disease (Figure S1). 


\section{Methods}

\section{Study recruitment and sample collection}

Non-smoking pregnant women $(n=138)$ with uncomplicated pregnancies undergoing elective terminations during mid-gestation of pregnancy (gestational week [GW] 15-24) were recruited at the Women's Options Center (WOC) in the San Francisco Bay Area from 2014 to 16. The WOC serves a racially/ethnically diverse low-income population of pregnant women in Northern and Central California who tend to rely on public health insurance coverage for prenatal care. Written and verbal consents were obtained during the clinical visit, followed by administration of a survey questionnaire to each study participant. All study protocols were approved by the UCSF Institutional Review Board prior to the clinical visit.

We collected the following biological samples-maternal serum, placenta, and fetal liver $(n=141$, due to three sets of twins) - from study participants during or immediately following the clinical procedure. Fetal sex was determined anatomically and recorded prior to the collection of fetal tissue samples. Chemical PBDE analyses were later performed on $n=135$ placental samples, with $n=130$ matched samples (excluding twins) of all three biomatrices (Fig. 2). For the chemical analyses, maternal serum samples were centrifuged at 3000 RPM for
$10 \mathrm{~min}$ at $4{ }^{\circ} \mathrm{C}$ prior to aliquoting and transfer of the serum with glass pipettes into pre-screened (to confirm absence of PBDE) sterilized amber vials (storage at $\left.80^{\circ} \mathrm{C}\right)$. A subset of placental samples $(n=62)$ was further processed and evaluated for three molecular and morphological features (see below). The placental subset was selected based on the highest and lowest PBDE exposure levels to maximize our ability to detect differences, which we expected would reduce selection bias and enhance generalizability across the whole population. Population characteristics between the subset assessed for placental biomarkers $(n=62)$ and the whole population $(n=130)$ were similar. Placental biopsies contained a section of the basal plate (which represents the maternal-fetal interface) and includes placental regions covered in our analysis (chorionic villi and decidua).

Placental tissues were submerged in medium (DME/ $\mathrm{H}-21$ [Gibco], 12.5\% fetal bovine serum [Hyclone], 1\% glutamine plus [Atlanta Biologicals], 1\% penicillin/ streptomycin [Invitrogen], and $0.1 \%$ gentamicin [Gibco]); dissected into $1 \mathrm{x} \mathrm{cm}^{3}$ pieces; fixed with $3 \%$ paraformaldehyde (PFA); and frozen in Optimal Cutting Temperature (OCT) medium (Sakura Finetek, SA62550-01) in a cryomold at $-80^{\circ} \mathrm{C}$ [42]. Placental biopsies were later sectioned ( $5 \mu \mathrm{M}$ thickness) using a

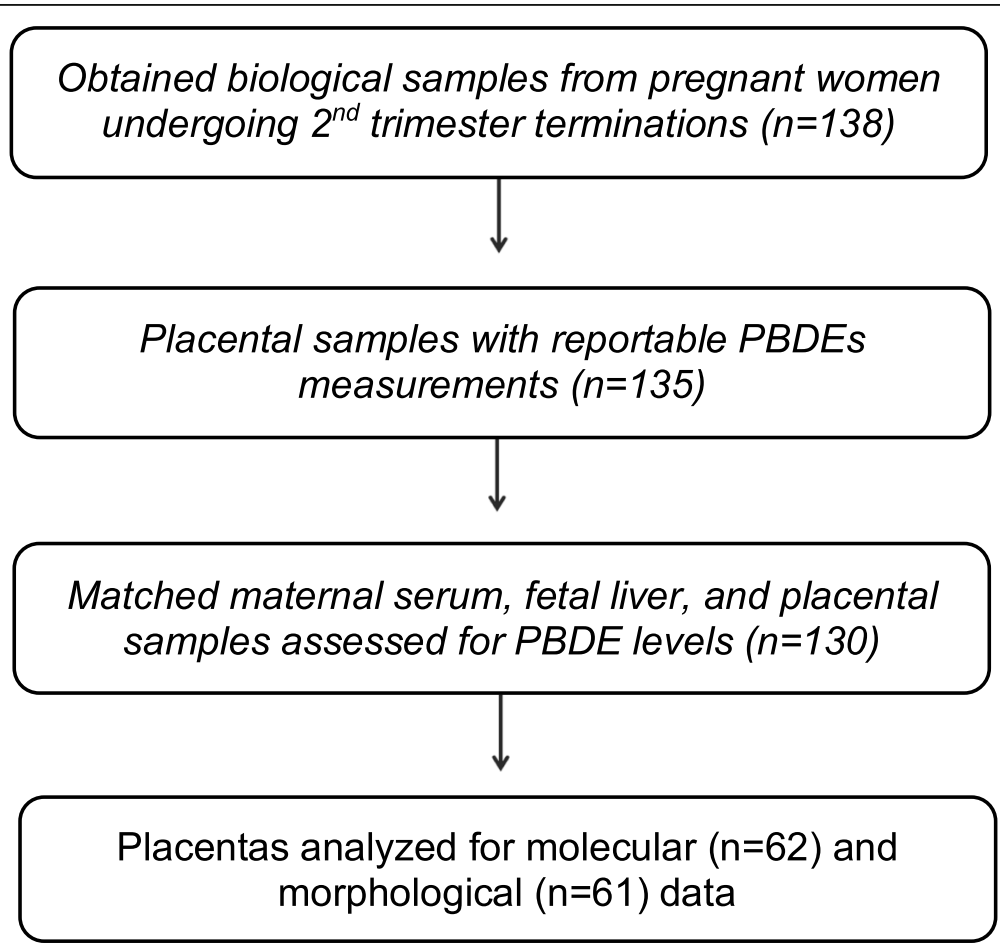

Fig. 2 Participant recruitment and biological samples collected at the Women's Options Center (WOC) in Northern California from 2014 to 16 . We obtained tissue samples and questionnaire data from 138 pregnant women undergoing elective terminations during mid-gestation.

Polybrominated diphenyl ether (PBDE) levels were measured in 135 placental samples. After excluding twins, PBDE measurements were obtained for 130 matched samples of maternal serum, fetal liver, and the placenta. Then we evaluated a subset of placental samples for molecular $(n=62)$ and morphological $(n=61)$ biomarkers of placental development and disease 
cryostat (Leica) and placed on glass slides for molecular and morphological assessments.

\section{Chemical exposure assessment}

Nineteen PBDE congeners (including BDE-17, -28, -47, $-66,-85,-99,-100,-153,-154,-183,-196,-197,-$ $201,-202,-203,-206,-207,-208$, and -209 ) were analyzed in maternal serum, placenta, and fetal liver by the Environmental Chemistry Laboratory at California's Department of Toxic Substances Control in Berkeley, CA, using gas chromatography-high-resolution mass spectrometry (GC-HRMS, DFS, ThermoFisher, Bremen, Germany). Isotopically-labeled internal surrogate mix standards (IS) were used for quantitation (Wellington Laboratories, Inc., Guelph, Ontario, Canada).

Thawed serum samples $(1 \mathrm{~mL})$ were spiked with carbon-labeled internal standards $\left({ }^{13} \mathrm{C}_{12}\right.$-BDE-28, 47, 99, $153,154,183,197,207$, and 209), with $4 \mathrm{~mL}$ each of formic acid and water added. Serum samples were vortexed and loaded into an automated sample extraction system (RapidTrace, Biotage; Uppsala Sweden). Oasis HLB cartridges ( 3 cc, $500 \mathrm{mg}$, Waters Corp.; Milford, MA) were used for sample extraction and acidified silica $\left(500{ }^{\circ} \mathrm{C}\right.$ prebaked, manually packed, $3 \mathrm{cc}$ ) for sample extraction cleanup. Final eluates were concentrated 10-fold using an automated nitrogen evaporation system (TurboVap LV, Biotage; Uppsala, Sweden) and spiked with a ${ }^{13} \mathrm{C}_{12^{-}}$ PCB-209 recovery standard [43, 44]. Total cholesterol and triglycerides were measured enzymatically by Boston Children's Hospital (Boston, MA) and subsequently used to calculate the total serum lipid concentration for each study participant using the Phillips formula [45]. Fetal liver and placental samples were analyzed using our liver analytical method with slight modification [25]. Before sample extraction, only placenta samples were lyophilized. Briefly, samples were homogenized and spiked with the same internal standards (listed above). Samples were then denatured with hydrochloric acid and extracted with 1:1 hexane:methyl tert-butyl ether (MTBE). Aqueous potassium chloride solution was added to each sample extract to remove potentially co-extracted aqueous compounds and the organic layer was re-extracted and dried in a pre-weighed, pre-baked aluminum weighing dish for lipid content determination via gravimetric analysis. Samples were then reconstituted in hexane and lipids were removed using concentrated sulfuric acid, followed by cleanup with acidified silica $\left(500^{\circ} \mathrm{C}\right.$ prebaked, manually packed, $3 \mathrm{cc}$ ) on automated SPE system (RapidTrace, Biotage; Uppsala Sweden).

\section{Assessment of stage-specific antigens and morphological endpoints}

Molecular and morphological placental features were evaluated independently by two investigators who were blinded to PBDE exposure levels. We assessed molecular immunoreactivity of CTBs at the maternal-fetal interface with antibodies specific for integrin alpha 1 (ITGA1), VE-cadherin (CDH5) or metalloproteinase 1 (MMP1) in five stages of trophoblast differentiation: Zone I) CTBs resident in FV; Zone II) CTBs in the proximal (p) regions of AV cell columns; Zone III) CTBs within the distal (d) regions of AV cell columns; Zone IV) interstitial invasive/extravillous CTBs (iCTB); and Zone V) endovascular CTBs (eCTB) (Fig. 1). We selected ITGA1 and $\mathrm{CDH} 5$ based on previous in vitro and in vivo literature demonstrating the importance of these adhesion receptor molecules in placental development and function (specifically regarding invasive and endovascular CTB differentiation pathways that are critical for vascular remodeling) as well as their potential disease associations. MMP1 is a metalloproteinase matrix-degrading enzyme that facilitates CTB migration/invasion during placentation $[7,9,46]$ (Table S1 and Figures S3, S4, S5). We immunolocalized ITGA1, CDH5, and MMP1 in each placental zone using published methods [47]. The antibodies and dilutions that were used for this purpose are described in (Table S2). CTBs were identified by reactivity with CK7. Batch effects which influence immunofluorescence (IF) intensity were continuously monitored by including serial sections from the same two placental samples in each assessment. Slides were imaged using an upright Leica DFC450 microscope equipped with a camera and Leica Advanced Fluorescence Application Suite Ver. 3.2 (Leica Microsystems). Immunoreactivity was scored using a previously established semi-quantitative approach in which two independent reviewers categorized immunofluorescence into three broad pre-defined categories (rather than continuous measures) based on the percentage of CTBs that reacted with each antibody: 1) $<25 \%(-)$; 2) 25-75\% (-/+); and 3) > 75\% (+) [47].

Placental biopsies were also stained with Hematoxylin and Eosin (H\&E) to evaluate morphological features: (1) average total number of white blood cells (WBC) present in basal plate (BP) per 10X field; (2) percent of FV with perivillous fibrinoid deposits $(\sim 60$ villi evaluated per field); 3) fibrinoid deposition at the utero-placental junction; and (4) total number of modulated uterine spiral arteries which contained CTBs (defined as presence of > $50 \%$ endovascular CTBs in the lining of uterine spiral arteries) (Table S1 and Figure S5). All values were based on examination of the entire placental section at $10 \mathrm{X}$ resolution $(\sim 6-10$ independent fields). In total, we evaluated 62 placentas; however, the sample size for each molecular and morphological endpoint varied depending on whether tissue sections captured the key structures of interest, most notably uterine arteries. For example, eCTB were confined to placental tissue sections that contained uterine arteries $(n=29-42) ; \mathrm{pAV}$ and dAV 
CTBs were observed in 51-53 samples, and iCTB were found in 60 samples (Table S3). For morphological assessments, one sample was lost while processing $(n=61$ placentas evaluated). Images were acquired using a bright field Leica DFC450 microscope equipped with a camera (Figure S5).

\section{Statistical analysis}

We calculated detection frequencies for all 19 PBDE congeners in matched samples of maternal serum, fetal liver, and placental tissues obtained in this study (201416; $n=130$ ) (Table S4). We normalized wet-weight PBDE concentrations to total lipid levels in order to account for possible measurement error related to estimating PBDE exposure from biomonitoring data, since biological proxies of exposure to PBDEs and other lipophilic compounds can vary systematically with population characteristics (e.g., lipid content) rather than the PBDE exposures they represent [48, 49]. For five PBDE congeners (BDE-28, - 47, - 99, - 100, and -153) detected in $>50 \%$ of placental samples $(n=135)$, we further calculated wet-weight and lipid-adjusted descriptive statistics, including geometric mean, interquartile range (25th-75th percentile), and range (min-max). We also computed a summary PBDE metric by adding four of congeners $(\Sigma \mathrm{PBDE} 4=\mathrm{BDE}-47,-99,-100$, and -153$)$ with detection frequencies $>50 \%$ in all maternal-fetal tissues, in order to facilitate results comparison with maternal serum and fetal liver in secondary analyses. We used maximum likelihood estimation (MLE) assuming a log-normal distribution to account for PBDE concentrations below the laboratory method detection limit (MDL) [50]. Statistical analyses were performed using $\mathrm{R}$ (Version 3.5.1) [51], with significance defined as $p<0.05$ and marginal significance as $p<0.10$ (two-sided tests).

We examined how PBDE concentrations varied across categorical population characteristics, including maternal age $(<20,20-24,25-29, \geq 30$ years), gestational age $(<$ $19,19-21, \geq 21$ weeks), body mass index (BMI; $<25,25-$ $30, \geq 30 \mathrm{~kg} / \mathrm{m} 2$ ), parity ( 0 or $\geq 1$ live births), fetal sex (male or female), education ( $\leq$ High School or $\geq$ Some college), type of insurance (public or private/self-pay), race/ethnicity (Latina/Hispanic, Non-Hispanic Black, Non-Hispanic White, Asian/Pacific Islander), birth country (U.S. or foreign born), and sample collection year $(2014,2015,2016)$. Although sample size precluded multivariable analysis, we examined population characteristics in separate models with our exposure (PBDE concentrations) and outcome of interest (placental biomarkers). For example, although fetal sex could conceivably be an effect modifier of PBDE effects on placental development and function, we did not observe variation with exposures or outcomes in this study.
In addition, we computed descriptive statistics for molecular and morphological placental biomarker data. We natural log-transformed average WBC counts prior to statistical analysis based on visual inspection of rightskewed distribution as well as results from the ShapiroWilk statistical test. One observation had a WBC count of zero which we set to 0.01 prior to log transformation. We calculated the percent of modulated uterine spiral arteries by dividing the number of uterine spiral arteries with $>50 \%$ endovascular CTB modulation by the total number of uterine spiral arteries observed. If the total number of uterine spiral arteries (i.e., the denominator) was zero or less than two, the denominator and percent modulation were re-coded as missing. Then we divided \% CTB-modulated uterine blood vessels $(\mathrm{mBV})$ at the median and categorized the variable into two groups (low and high). We modeled molecular immunoreactivity categorically rather than continuously based on the three pre-defined categories that were scored by two independent reviewers in the laboratory (as described in section 2.3$)$ : $(1)<25 \%(-)$; 2) $25-75 \%(-/+)$; and 3$)>$ $75 \%(+)$.

Kendall's Tau Correlation Coefficient [52], a nonparametric measure of correlation that compares concordant and discordant pairs of ordered data to determine the extent of increasing or decreasing covariation between two variables, was used to evaluate monotonic relationships: 1) among molecular and morphological placental biomarkers; and 2) between placental biomarkers (molecular and morphological) and placental PBDE concentrations (wet-weight and lipid-normalized). We calculated the correlation between placental biomarkers and PBDE concentrations using the censored version of Kendall's tau [53]. We adjusted for multiple comparisons by estimating the false discovery rate (FDR) using the Benjamini and Hochberg method [54].

To compare PBDE levels across immunoreactivity groups, we tested group mean differences (assuming a lognormal PBDE distribution) using MLE to account for leftcensored PBDE data [50]. From these bivariate censored regression/ANOVA models, we calculated the pairwise percent (\%) differences in PBDE concentrations as $\left(\mathrm{e}^{\beta}-1\right)$ * 100 and the $95 \%$ confidence interval $(95 \% \mathrm{CI})$ as $\left(\mathrm{e}^{\beta \pm 1.96 \times}\right.$ $\mathrm{SE}-1) * 100$, where the referent group comprised placental samples in the $<25 \%(-)$ immunoreactivity group. The beta coefficient from these models represents the difference in PBDE levels on the lognormal scale between moderate or high immunoreactivity groups and the referent group, while $\mathrm{e}^{\beta}$ represents the ratio of average PBDE concentrations between immunoreactivity groups. We also used the Fisher's exact test to examine whether molecular immunoreactivity varied by high/low PBDE exposure (where PBDE levels were divided at the median and categorized into high vs. low exposure groups). 
In addition to assessing correlations between placental PBDE concentrations and placental biomarkers, we also re-examined placental biomarker associations with fetal liver and maternal serum PBDE concentrations, since we previously found moderate correlation between congeners across maternal-fetal tissues during mid- gestation, with cross-tissue correlations ranging from 0.18 (BDE-99) to $\geq 0.50$ (BDE-47, -100 , and - 153) $(p<0.0001)$ [55].

\section{Results}

The majority of women in our study population $(n=$ 135 ) were $<30$ yrs. old (79\%); participants $20-24$ yrs. old were the largest age subgroup (43\%; Table 1). Gestational age ranged from $\sim 15-24$ wks (median $=20 \mathrm{wks}$ ). Most women were classified as overweight (28\%; BMI = $\left.25-30 \mathrm{~kg} / \mathrm{m}^{2}\right)$ or obese (29\%; BMI $\left.\geq 30 \mathrm{mg} / \mathrm{m}^{2}\right)$. Approximately $60 \%$ of participants had at least one prior live birth and $54 \%$ of the pregnancies were with a male fetus. Education level was evenly divided between high school attendance or graduation and some post-secondary schooling. Most women used public rather than private health insurance. The study population was racially/ethnically diverse (> 50\% Latina/Hispanic and non-Hispanic Black) (Table 1).

PBDEs were detected in all placental samples, with the range of wet-weight PBDE concentrations spanning more than one order of magnitude, and lipid-adjusted PBDE levels greater than wet-weight concentrations by almost two orders of magnitude (Table 1). Placental PBDE concentrations did not vary by maternal age, gestational age, BMI, parity, fetal sex, education, or insurance type $(p>0.05)$. In contrast, we observed placental PBDE exposure differences based on race/ethnicity, birth country, and sample collection year. More specifically, placental PBDE concentrations were highest among USborn participants, Non-Hispanic Black women, and in earlier collection years $(2016>2014>2015)$ (Table 1$)$, which is consistent with previous findings although birth country differences should be interpreted in the context of missing data for most study participants [55].

ITGA1, CDH5, and MMP1 immunoreactivity was in accord with previously published results [7, 9, 46]. These stage-specific antigens were upregulated as CK-positive CTBs exited the placenta and invaded the uterine wall $(p<0.001)$ (Fig. 3 and Table S3). Staining patterns varied by region and antibody target. For example, ITGA1 immunoreactivity was minimally detected in FV CK+ CTBs and upregulated as the cells differentiated and invaded, increasingly detected in association with $\mathrm{pAV}, \mathrm{dAV}$, iCTB, and eCTB CTBs. CDH5 immunoreactivity had a similar pattern except that FV CK+ CTBs in a few samples expressed this cell-cell adhesion molecule. With the exception of eCTB, MMP1 was more widely expressed by the CTB subpopulations on which we focused.
On average, WBC infiltration was 212 cells \pm 174 (Table 2). The percentage of FV with associated fibrin deposits was $13.4 \pm 10.4 \%$, while fibrinoid deposition in the BP delimited $22.6 \pm 19.6 \%$ of the CTB-decidual boundary. In a subset of placental biopsies $(n=38)$ with at least three uterine spiral arteries, $71.7 \pm 30.4 \%$ of blood vessels were modulated, defined as having $>50 \%$ CK-positive CTBs within the walls or lumens. As expected, given the narrow range in gestational age, correlations between morphological features and weeks of pregnancy were not significant. The exception was possible evidence of an inverse relationship between perivillous fibrinoid deposition and gestational age $(\tau=-0.28$; $p=0.001$ ) (Table 2).

The rank order correlation of ITGA1, CDH5, and MMP1 immunoreactivity varied among molecules and placental region/cell type (Tables S5 and S6). Molecular markers were more highly correlated between CTB subtypes that were closely related on a developmental continuum and located in proximity to one another (iCTB and eCTB; pAV and dAV). For example, previous studies showed that the stage-specific antigens of interest are upregulated as the cells leave the placenta and invade the uterine wall $[7,9,46]$. Accordingly, MMP1 immunoreactivity in $\mathrm{CK}+$ cells was positively correlated between CTBs in pAV and dAV $(\tau=0.42)$, dAV CTBs and iCTB $(\tau=0.46)$, and $\mathrm{iCTB}$ and $\mathrm{eCTB}(\tau=0.32)$; there was no correlation between CTBs in FV and pAV. As expected, we found ITGA1 and CDH5 were similarly regulated. Interestingly, while we found no-to-low correlation between ITGA1 and CDH5 immunoreactivity, regardless of placental region/cell type, MMP1 immunoreactivity was modestly correlated with the other two molecular markers. We found little association among the morphological endpoints except for a positive correlation between fibrinoid deposition in the FV and basal plate ( $\tau=$ $0.24, p=0.01)$. In the absence of additional data, we were unable to determine whether strong correlations exist between molecular and morphological biomarkers, although this analysis was limited by sample size. However, several trends were notable, including a consistent inverse correlation between immunoreactivity for MMP1 $(\tau=-0.27)$, ITGA1 $(\tau=-0.21)$, and CDH5 ( $\tau=$ - 0.26) in dAV and WBC count. There was also an inverse association between expression of these stage specific antigens and fibrinoid deposition in some regions. For example, reduced MMP1 immunoreactivity ( $\tau=-$ 0.29) in CTBs of the dAV and the percent of floating villi with fibrinoid deposits and vice versa. Adjusting for multiple comparisons did not substantially change overall correlation patterns between molecular and morphological biomarkers (Tables S5 and S6).

In general, we did not observe significant correlations between PBDE concentrations (BDE-47 or $\Sigma$ PBDEs) and 
Table 1 Population characteristics and placental PBDE levels during mid-gestation at the Women's Options Center (WOC) in Northern California, 2014-16 $(n=135)$

\begin{tabular}{|c|c|c|c|c|c|c|c|c|c|}
\hline \multirow[b]{2}{*}{ Population Characteristic } & \multirow[b]{2}{*}{$n(\%)$} & \multicolumn{2}{|l|}{ Wet-weight BDE-47 } & \multicolumn{2}{|c|}{ Lipid-adjusted BDE-47 } & \multicolumn{2}{|c|}{ Wet-weight $\sum$ PBDE4 ${ }^{a}$} & \multicolumn{2}{|c|}{$\overline{\text { Lipid-adjusted } \sum P B D E 4^{a}}$} \\
\hline & & $\overline{G M}(95 \%$ Cl) & $p$-Value $e^{b}$ & $\overline{G M(95 \% C l)}$ & $p$-Value ${ }^{b}$ & $\overline{G M}(95 \% C l)$ & $p$-Value ${ }^{b}$ & $\overline{G M}(95 \% C l)$ & $p$-Value ${ }^{b}$ \\
\hline \multicolumn{10}{|l|}{ Maternal Age } \\
\hline$<20$ years & $19(14)$ & $0.10(0.08,0.14)$ & 0.910 & $11.1(8.4,14.6)$ & 0.945 & $0.20(0.16,0.26)$ & 0.922 & $21.6(16.9,27.5)$ & 0.893 \\
\hline 20-24 years & $58(43)$ & $0.10(0.08,0.14)$ & & $10.8(7.8,14.9)$ & & $0.20(0.15,0.27)$ & & $20.9(15.7,27.8)$ & \\
\hline $25-29$ years & $30(22)$ & $0.10(0.07,0.14)$ & & $11.1(7.7,15.8)$ & & $0.20(0.14,0.27)$ & & $22.1(16.1,30.4)$ & \\
\hline$\geq 30$ years & $29(21)$ & $0.10(0.07,0.15)$ & & $11.3(7.8,16.2)$ & & $0.19(0.14,0.26)$ & & $21.0(15.3,29.0)$ & \\
\hline \multicolumn{10}{|l|}{ Gestational Age } \\
\hline$<19$ weeks & $37(27)$ & $0.11(0.09,0.13)$ & 0.255 & $11.6(9.5,14.1)$ & 0.601 & $0.21(0.18,0.25)$ & 0.440 & $22.4(18.8,26.8)$ & 0.756 \\
\hline 19-21 weeks & $51(38)$ & $0.11(0.09,0.14)$ & & $11.3(8.7,14.8)$ & & $0.21(0.16,0.26)$ & & $21.3(16.8,26.9)$ & \\
\hline$\geq 21$ weeks & $48(35)$ & $0.09(0.07,0.12)$ & & $10.2(7.8,13.4)$ & & $0.18(0.15,0.23)$ & & $20.5(16.2,26.0)$ & \\
\hline \multicolumn{10}{|l|}{ Body Mass Index (BMI) } \\
\hline $\begin{array}{l}\text { Normal/Under }(<25 \mathrm{~kg} / \\
\left.\mathrm{m}^{2}\right)\end{array}$ & $59(43)$ & $0.10(0.08,0.11)$ & 0.602 & $10.8(9.2,12.7)$ & 0.795 & $0.19(0.16,0.22)$ & 0.594 & $21.1(18.3,24.3)$ & 0.652 \\
\hline $\begin{array}{l}\text { Overweight (25-30 kg/ } \\
\left.\mathrm{m}^{2}\right)\end{array}$ & $38(28)$ & $0.11(0.09,0.14)$ & & $10.6(8.1,13.7)$ & & $0.21(0.17,0.26)$ & & $20.2(16.1,25.5)$ & \\
\hline Obese $\left(\geq 30 \mathrm{~kg} / \mathrm{m}^{2}\right)$ & $39(29)$ & $0.10(0.08,0.13)$ & & $11.6(9.0,15.0)$ & & $0.20(0.16,0.25)$ & & $22.7(18.2,28.4)$ & \\
\hline \multicolumn{10}{|l|}{ Parity } \\
\hline 0 live births & $55(40)$ & $0.09(0.08,0.11)$ & 0.309 & $9.9(8.4,11.6)$ & 0.240 & $0.19(0.16,0.21)$ & 0.443 & $19.6(16.9,22.6)$ & 0.325 \\
\hline$\geq 1$ live births & $81(60)$ & $0.11(0.09,0.13)$ & & $11.9(9.6,14.7)$ & & $0.21(0.17,0.25)$ & & $22.6(18.7,27.4)$ & \\
\hline \multicolumn{10}{|l|}{ Fetal Sex } \\
\hline Male & $73(54)$ & $0.10(0.09,0.12)$ & 0.955 & $10.2(8.8,11.8)$ & 0.379 & $0.20(0.18,0.23)$ & 0.942 & $20.4(18.0,23.3)$ & 0.659 \\
\hline Female & $63(46)$ & $0.10(0.09,0.13)$ & & $11.9(9.6,14.7)$ & & $0.20(0.16,0.23)$ & & $22.3(18.5,26.9)$ & \\
\hline \multicolumn{10}{|l|}{ Education } \\
\hline$\leq$ High School & $67(49)$ & $0.11(0.10,0.13)$ & 0.260 & $11.9(10.2,13.8)$ & 0.415 & $0.22(0.19,0.25)$ & 0.177 & $23.1(20.2,26.3)$ & 0.308 \\
\hline$\geq$ Some college & $68(50)$ & $0.09(0.08,0.12)$ & & $10.3(8.3,12.7)$ & & $0.18(0.15,0.22)$ & & $19.9(16.5,24.0)$ & \\
\hline \multicolumn{10}{|l|}{ Insurance } \\
\hline Public & $98(72)$ & $0.11(0.10,0.12)$ & 0.364 & $11.7(10.3,13.3)$ & 0.159 & $0.21(0.19,0.23)$ & 0.222 & $22.7(20.4,25.3)$ & 0.066 \\
\hline Private/Self-pay & $37(27)$ & $0.09(0.07,0.11)$ & & $9.3(7.3,11.7)$ & & $0.17(0.14,0.21)$ & & $17.7(14.4,21.8)$ & \\
\hline \multicolumn{10}{|l|}{ Race/ethnicity } \\
\hline Latina/Hispanic & $45(33)$ & $0.10(0.09,0.12)$ & 0.102 & $10.2(8.6,12.1)$ & 0.038 & $0.19(0.16,0.22)$ & 0.011 & $18.8(16.2,21.8)$ & 0.001 \\
\hline Non-Hispanic Black & $31(23)$ & $0.12(0.09,0.16)$ & & $13.7(10.4,17.9)$ & & $0.24(0.19,0.31)$ & & $27.6(21.9,34.8)$ & \\
\hline Non-Hispanic White & $20(15)$ & $0.09(0.07,0.13)$ & & $10.6(7.8,14.4)$ & & $0.18(0.14,0.23)$ & & $20.2(15.4,26.3)$ & \\
\hline Asian/Pacific Islander & $17(12)$ & $0.09(0.06,0.12)$ & & $9.2(6.6,12.7)$ & & $0.16(0.12,0.21)$ & & $17.0(12.8,22.6)$ & \\
\hline \multicolumn{10}{|l|}{ Birth Country } \\
\hline U.S. born & $71(52)$ & $0.11(0.10,0.13)$ & 0.114 & $11.8(10.3,13.6)$ & 0.222 & $0.21(0.19,0.23)$ & 0.009 & $22.4(19.9,25.3)$ & 0.025 \\
\hline Foreign born & $9(7)$ & $0.07(0.05,0.11)$ & & $8.3(5.5,12.4)$ & & $0.12(0.08,0.17)$ & & $13.5(9.4,19.3)$ & \\
\hline \multicolumn{10}{|l|}{ Collection Year } \\
\hline 2014 & $33(24)$ & $0.11(0.09,0.13)$ & 0.032 & $13.8(11.3,16.9)$ & 0.004 & $0.20(0.17,0.24)$ & 0.125 & $24.8(20.7,29.7)$ & 0.037 \\
\hline 2015 & $93(68)$ & $0.10(0.08,0.12)$ & & $9.8(7.7,12.3)$ & & $0.19(0.16,0.24)$ & & $19.6(15.9,24.3)$ & \\
\hline 2016 & $10(7)$ & $0.16(0.10,0.26)$ & & $15.9(10.0,25.3)$ & & $0.29(0.19,0.43)$ & & $28.0(18.4,42.5)$ & \\
\hline Total (pooled) $)^{b}$ & $135(100)$ & $0.10(0.09 ; 0.03-0.92)$ & & $\begin{array}{l}11.0(9.7 ; 3.1- \\
107)\end{array}$ & & $\begin{array}{l}0.20(0.15 ; 0.07- \\
1.3)\end{array}$ & & $\begin{array}{l}21.3(16.4 ; 5.8- \\
152)\end{array}$ & \\
\hline
\end{tabular}

GM Geometric mean, IQR Interquartile range (25th-75th percentile)

† Number (\%) missing $=56(41), 1(0.7), 1(0.7)$, and 23 (17) for birth country, education, insurance, and race/ethnicity, respectively

${ }^{a} \sum$ PBDE4 $=B D E-47+B D E-99+B D E-100+B D E-153$

${ }^{b} P$-values from ANOVA test of mean difference in Ln PBDE levels between population characteristic subgroups. $P<0.10$ bolded

'Summary statistics of placental PBDE levels in total (pooled) study population, including: GM (IQR; Min-Max) 
most molecular or morphological biomarkers (Table S7). The exception was an inverse relationship between ITGA1 immunoreactivity in eCTB with levels of most PBDE congeners, individually and as a group. For example, BDE-47 was inversely correlated with eCTB ITGA1 $(\tau=-0.25)$. Similar inverse relationships were observed for congeners $-99,-100$, and $\Sigma$ PBDE4 but were attenuated for BDE-28 and BDE-153. We also found a positive correlation between $\mathrm{BDE}-28$ and MMP1 immunoreactivity in $\mathrm{iCTB}(\tau=0.18)$. Correlations with lipid-adjusted PBDE concentrations were similar and thus not reported. Adjusting for multiple comparisons revealed the potential for false positive findings (Table S7), suggesting these observations could be strengthened by analyzing more samples.

The analysis revealed a similar inverse trend in the magnitude of ITGA1 immunoreactivity and placental concentrations of PBDE congeners. For example, placentas with the highest levels of ITGA expression in eCTB had $42 \%$ (95\% CI: $-69-7.2 \%$ ) lower levels of $\Sigma$ PBDE4 compared to the referent group (Table 3 and Fig. 4). The magnitude and direction of association with CTBe ITGA-1 immunoreactivity was consistent for all congeners, despite ANOVA tests which did not reveal an overall difference between groups $(p>0.05)$. We also found a positive association between wet-weight and lipid-adjusted placental BDE-47 or $\Sigma$ PBDE4 concentrations and CDH5 immunoreactivity in $\mathrm{iCTB}(p<0.05)$.
The same relationship held for $\mathrm{eCTB}$, although at a lower level of statistical significance (Table 3 and Fig. 4).

We observed a similar pattern of association with PBDEs in maternal serum and the fetal liver, with ITGA1 (eCTB) and CDH5 (iCTB) correlations that were strongest in the fetal liver, and minimal evidence of correlation with morphological biomarkers (Supplemental Spreadsheet).

\section{Discussion}

This is the largest study to our knowledge which characterizes a combination of molecular and morphological biomarkers measured with high resolution and precision directly in placental tissues during the second trimester. We additionally examined their relationship to placental (and maternal-fetal) PBDE concentrations for a first ever in vivo investigation of PBDEs and mechanistic effects on placentation during a vulnerable window of development. We immunolocalized for three molecules-integrin alpha 1 (ITGA1), VE-cadherin (CDH5), and metalloproteinase 1 (MMP1)-in CTBs within six regions of the basal plate and confirmed their increased presence as they invaded into the maternal unit. We also found possible inverse and positive PBDE associations with ITGA1 immunoreactivity (endovascular CTBs) and CDH5 immunoreactivity (interstitial and endovascular CTBs), respectively, which are molecular hallmarks of placental development and function during pregnancy.

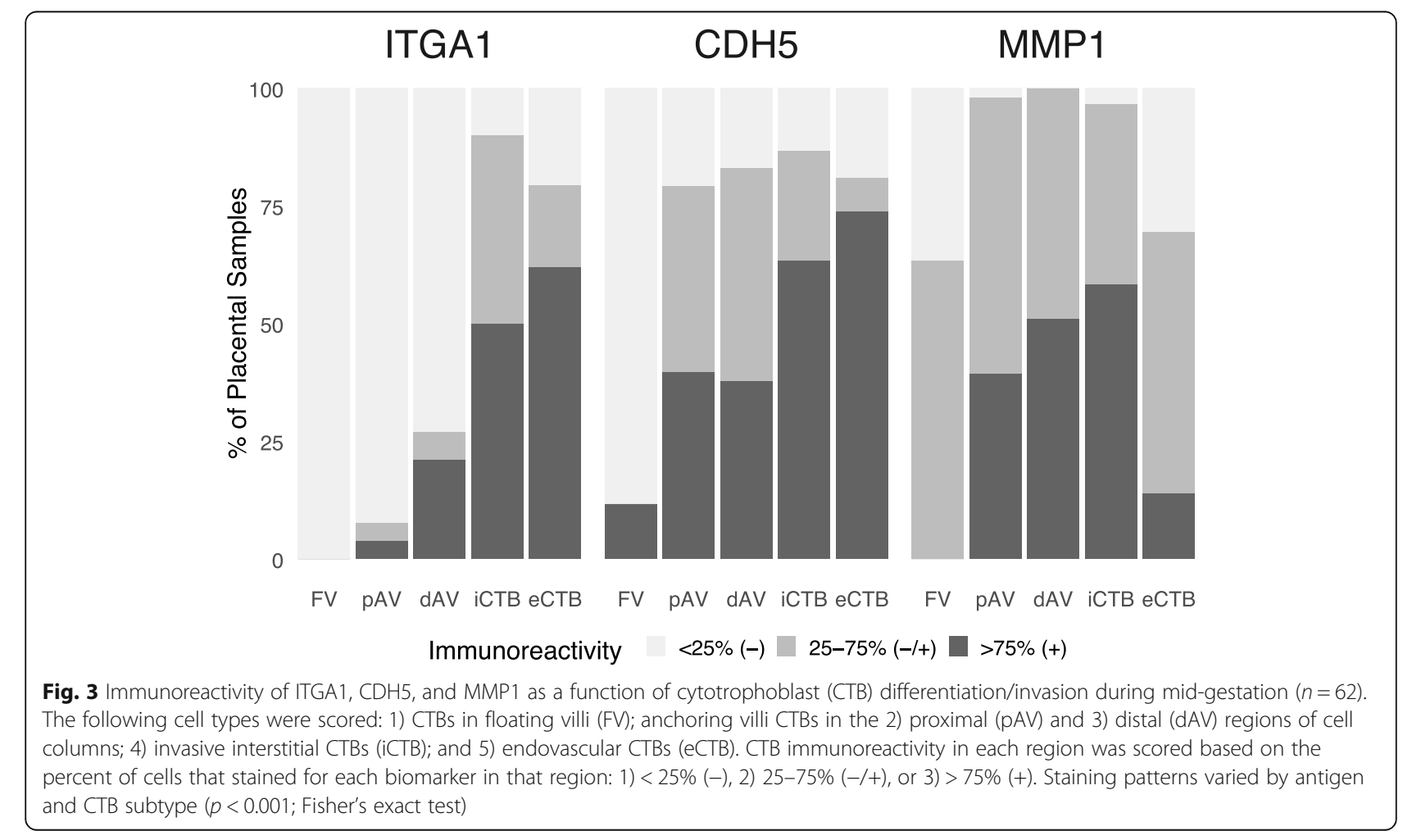


Table 2 Descriptive statistics of morphological placental biomarkers and correlation ${ }^{a}$ with gestational age during mid-gestation $(n=61)$

\begin{tabular}{lllllllll}
\hline Morphological Biomarker & & & & & & \multicolumn{2}{c}{ Gestational Age (Weeks) } \\
\cline { 6 - 10 } & $\boldsymbol{n}$ & Mean & Median & SD & Min & Max & Tau & p-Value \\
\hline Average White Blood Cell / image & 61 & 212 & 157 & 174 & 0.00 & 950 & -0.12 & 0.179 \\
\% Floating Villi with Fibrinoid Deposition (binary) & 61 & 13.4 & 11.8 & 10.4 & 0.10 & 47.1 & -0.28 & 0.001 \\
\% Fibrinoid Deposition in Basal Plate (area) & 61 & 22.6 & 20.0 & 19,6 & 0.00 & 78.0 & 0.02 & 0.866 \\
\% CTB-modulated Uterine Spiral Arteries & 38 & 71.7 & 80.6 & 30.4 & 0.00 & 100 & -0.14 & 0.240 \\
\hline
\end{tabular}

CTB Cytotrophoblast, SD Standard deviation

${ }^{a}$ Non-parametric rank order correlation assessed using Kendall's Tau Correlation Coefficient

ITGA1 and CDH5 play key roles in CTB differentiation and have potential disease associations (e.g., preeclampsia) $[7,9,11]$. We also selected to examine MMP1 in vivo since our previous in vitro analysis revealed upregulated expression of $M M P 1$ in differentiating CTBs exposed to BDE-47 [40]. In agreement with previously published data [42], ITGA1 was upregulated in cell columns and primarily expressed in extravillous CTBs, especially in the interstitial and endovascular regions of the basal plate (iCTB and eCTB). Corresponding with previous observations [7], $\mathrm{CDH} 5$ had a similar expression pattern. MMP1 is thought to be involved in extracellular matrix (ECM) breakdown during CTB invasion [56]. We confirmed upregulated anti-MMP1 reactivity associated with CTBs of the cell column, iCTB, and eCTB, Thus, the stage-specific antigens we profiled are upregulated as part of the molecular program that enables placental uterine attachment and arterial remodeling. Motivated by our in vitro exposure data showing that BDE-47 inhibits CTB migration and invasion, we further investigated whether PBDE exposures in vivo were associated with reduced immunoreactivity of molecules that are typically upregulated during these processes. The association between decreased ITGA1 expression in endovascular CTBs and high levels of PBDE exposures was consistent with this hypothesis. However, we also observed a positive association between these exposures and enhanced immunoreactivity for CDH5 in invasive CTBs (interstitial and endovascular), for which there may be several possible explanations, given the large constellation of molecules (including transcription factors, adhesion molecules, and proteinases) and inhibitors that interact during placental invasion and vascular remodeling.

The exact mechanisms and molecular interactions during placentation remain unresolved, leaving many potential reasons for the discordant PBDE results between ITGA1 and CDH5. Compensatory mechanisms, other environmental contributors [45], developmental and localized timing of the expression of ITGA1/CDH5, and human variability may also influence the effect of PBDE exposure on the presence of ITGA1/CDH5 in invading populations of CTBs. For example, upregulated
$\mathrm{CDH} 5$, which is an endothelial specific adhesion molecule expressed by CTBs as they migrate towards the uterus and translocate to the endothelial-lined maternal blood vessels [57], may be compensating for downregulated ITGA1, which is thought to help regulate the depth of trophoblast invasion [9]. PBDEs can also disrupt other pathways that regulate CTB differentiation, including hormone signaling, oxidative stress, inflammation, and placental developmental pathways [31, 33, 35, 40, 41], however, the direct interactions leading to increased $\mathrm{CDH} 5$ expression during CTB invasion is currently unknown and speculative in the context of environmental exposures. Moreover, we did not account for exposure to other chemicals such as Bisphenol-A (BPA), an endocrine disrupting compound that promotes trophoblast migration and disrupts placentation in mice by increasing the expression of invasionpromoting integrins (i.e., ITGB2 and ITGA5) and metalloproteinases (i.e., MMP-2 and MMP-9) [58]. Finally, despite the relatively large sample size of our study, variability was limited since we could not always sample in every placental region. Nevertheless, while the molecular complexity of CTB invasion presents challenges for predicting outcomes of biomarker experiments in the context of the exposome, future work linking PBDE and other chemical exposures with well-studied molecular measures of placenta-mediated pregnancy complications (i.e., CDH5 and ITGA1) and other sensitive indicators of placental development and disease would strengthen epidemiologic research on chemical exposures and placenta-mediated adverse health outcomes.

As to the MMP1 analyses, the effects of in vitro PBDE exposures were analyzed at the RNA level [40] as compared to the antibody-based protein approach we employed. Lack of correlation between transcriptomic and proteomic data is now a well-recognized phenomenon [59] and the effects of acute (in vitro) vs. chronic (in vivo) PBDE exposures are not well understood. Moreover, subtle effects from chemical exposures are difficult to detect in smaller epidemiologic studies (due to substantial human variability that can diminish statistical power). Additional biomarker studies with environmental chemicals that may have stronger effects on placental development, such as BPA, per- and 
Table 3 Percent (\%) difference in placental PBDE levels between molecular immunoreactivity groups for each biomarker within each placental region/cell type during mid-gestation $(n=62)$

\begin{tabular}{|c|c|c|c|c|c|c|c|c|c|}
\hline \multirow[b]{2}{*}{ Immunoreactivity } & \multirow[b]{2}{*}{$n$} & \multicolumn{2}{|c|}{ Wet-weight BDE-47 (ng/g) } & \multicolumn{2}{|c|}{ Wet-weight ¿PBDE4 (ng/g) } & \multicolumn{2}{|c|}{ Lipid-adjusted BDE-47 (ng/g lipid) } & \multicolumn{2}{|c|}{ Lipid-adjusted $\sum$ PBDE4 (ng/g lipid) } \\
\hline & & $\% \operatorname{diff}\left(95 \%(\mathrm{Cl})^{a}\right.$ & $p$-value & $\% \operatorname{diff}\left(95 \%(\mathrm{Cl})^{a}\right.$ & $p$-value ${ }^{b}$ & $\% \operatorname{diff}(95 \% \mathrm{Cl})^{a}$ & $p$-value ${ }^{b}$ & $\% \operatorname{diff}(95 \% \mathrm{Cl})^{a}$ & $p$-value ${ }^{b}$ \\
\hline \multicolumn{10}{|c|}{ Anchoring Villi (proximal) } \\
\hline $\mid T G A 1<25 \%(-)$ & 48 & Referent & 0.616 & Referent & 0.433 & Referent & 0.813 & Referent & 0.724 \\
\hline $25-75 \%(-/+)$ & 2 & $68(-44,403)$ & & $82(-32,385)$ & & $38(-56,331)$ & & $49(-46,316)$ & \\
\hline$>75 \%(+)$ & 2 & $-16 .(-72,151)$ & & $-21(-70,110)$ & & $23(-61,283)$ & & $15(-59,221)$ & \\
\hline CHD5 $<25 \%(-)$ & 11 & Referent & 0.502 & Referent & 0.404 & Referent & 0.441 & Referent & 0.357 \\
\hline $25-75 \%(-/+)$ & 21 & $35(-20,130)$ & & $39(-14,125)$ & & $39(-20,142)$ & & $43(-13,136)$ & \\
\hline$>75 \%(+)$ & 21 & $13(-34,91)$ & & $21(-25,94.8)$ & & $11(-36,93)$ & & $19(-28,96)$ & \\
\hline$M M P 1<25 \%(-)$ & 1 & Referent & 0.395 & Referent & 0.472 & Referent & 0.741 & Referent & 0.862 \\
\hline $25-75 \%(-/+)$ & 30 & $109(-51,797)$ & & $56(-58,481)$ & & $69(-64,700)$ & & $26(-69,413)$ & \\
\hline$>75 \%(+)$ & 20 & $67(-61,626)$ & & $2(-6,375)$ & & $51(-69,627)$ & & $14(-72,371)$ & \\
\hline \multicolumn{10}{|c|}{ Anchoring Villi (distal) } \\
\hline $\mid T G A 1<25 \%(-)$ & 38 & Referent & 0.759 & Referent & 0.837 & Referent & 0.599 & Referent & 0.739 \\
\hline $25-75 \%(-/+)$ & 3 & $-19(-68,102)$ & & $4.1(-54,138)$ & & $-28(-72,83)$ & & $-7.8(-61,116)$ & \\
\hline$>75 \%(+)$ & 11 & $15(-32,94)$ & & $15(-28,85)$ & & $19(-30,104)$ & & $20(-26,95)$ & \\
\hline CHD5 < 25\% (-) & 9 & Referent & 0.670 & Referent & 0.550 & Referent & 0.662 & Referent & 0.542 \\
\hline $25-75 \%(-/+)$ & 24 & $29(-26,125)$ & & $32(-20,120)$ & & $31(-27,135)$ & & $35(-21,128)$ & \\
\hline$>75 \%(+)$ & 20 & $17(-34,108)$ & & $19(-29,100)$ & & $19(-35,117)$ & & $21(-30,108)$ & \\
\hline MMP1 < 25\% (-) & 0 & Referent & 0.901 & Referent & 0.944 & Referent & 0.661 & Referent & 0.697 \\
\hline $25-75 \%(-/+)$ & 25 & $-9.1(-40,37)$ & & $-6.2(-35,36)$ & & $-18(-47,26)$ & & $-15(-43,24)$ & \\
\hline$>75 \%(+)$ & 26 & - & & - & & $0.0(0.0,0.0)$ & & $0.0(0.0,0.0)$ & \\
\hline \multicolumn{10}{|l|}{ Interstitial CTB } \\
\hline $\mid T G A 1<25 \%(-)$ & 6 & Referent & 0.622 & Referent & 0.556 & Referent & 0.881 & Referent & 0.863 \\
\hline $25-75 \%(-/+)$ & 24 & $-16(-58,66)$ & & $-17.1(-55,53)$ & & $-3.5(-53,98)$ & & $-4.4(-49,81)$ & \\
\hline$>75 \%(+)$ & 30 & $2.3(-48,101)$ & & $1.07(-45,85)$ & & $7.6(-47,117)$ & & $6.3(-43,99)$ & \\
\hline CHD5 < 25\% (-) & 8 & Referent & $0.032^{* *}$ & Referent & $0.028^{* *}$ & Referent & $0.018^{* *}$ & Referent & $0.019 * *$ \\
\hline $25-75 \%(-/+)$ & 14 & $138(26,350)^{* * *}$ & & $115(22,279)^{* * *}$ & & $163(37,405)^{* * *}$ & & $137(33,325)^{* * *}$ & \\
\hline$>75 \%(+)$ & 38 & $63(-6.6,185)^{*}$ & & $42(-14,133)$ & & $102(14,257)^{* *}$ & & $75(5.3,192)^{* *}$ & \\
\hline MMP1 < 25\% (-) & 2 & Referent & 0.372 & Referent & 0.350 & Referent & 0.693 & Referent & 0.727 \\
\hline $25-75 \%(-/+)$ & 23 & $41(-53,324)$ & & $-0.38(-63,167)$ & & $36(-57,330)$ & & $-4.1(-66,168)$ & \\
\hline$>75 \%(+)$ & 35 & $77(-40,425)$ & & $29(-51,242)$ & & $53(-51,376)$ & & $12(-59,208)$ & \\
\hline \multicolumn{10}{|l|}{ Endovascular CТВ } \\
\hline $\mid T G A 1<25 \%(-)$ & 6 & Referent & 0.303 & Referent & 0.211 & Referent & 0.518 & Referent & 0.386 \\
\hline $25-75 \%(-/+)$ & 5 & $-30(-73,80)$ & & $-22(-65,73)$ & & $-9.3(-66,145)$ & & $1.0(-57,135)$ & \\
\hline$>75 \%(+)$ & 18 & $-44(-73,16)$ & & $-42(-69,7.2)^{*}$ & & $-34(-69,44)$ & & $-31(-64,32)$ & \\
\hline CHD5 < 25\% (-) & 8 & Referent & 0.256 & Referent & 0.175 & Referent & 0.145 & Referent & 0.151 \\
\hline $25-75 \%(-/+)$ & 3 & $140(-16,590)$ & & $146(-2.8,521)^{*}$ & & $134(-21,588)$ & & $139(-8.3,522)^{*}$ & \\
\hline$>75 \%(+)$ & 31 & $42(-24,162)$ & & $23(-29,112)$ & & $82.4(-3.2,243)$ & & $59(-9.5,178)$ & \\
\hline MMP1 < 25\% (-) & 11 & Referent & 0.883 & Referent & 0.846 & Referent & 0.847 & Referent & 0.736 \\
\hline $25-75 \%(-/+)$ & 20 & $12(-40,110)$ & & $1.1(-42,77)$ & & $8.0(-43,106)$ & & $-2.8(-45,73)$ & \\
\hline$>75 \%(+)$ & 5 & $-6.6(-6,130)$ & & $-19(-64,83)$ & & $-16(-67,113)$ & & $-27(-68,67)$ & \\
\hline
\end{tabular}

${ }^{*} p<0.10 .{ }^{* *} p<0.05$. ${ }^{* *} p<0.01$. Significant and marginally significant results bolded

${ }^{a}$ Calculated from bivariate censored regression models, where referent group included placental samples with trophoblast cells (identified as cytokeratin+ cells) that did not stain (-) or stained only minimally $(0-25 \%)$ for antigen specific antibodies corresponding to each molecular biomarker

${ }^{b} P$-value from ANOVA test of mean difference in Ln PBDE level across three immunoreactivity groups 
poly-fluorinated compounds (PFAS), and heavy metals, could potentially highlight the utility of MMP1 and other potential molecular biomarker candidates in future studies. Nevertheless, our use of high resolution and high precision measures can generate useful insights even in smaller epidemiologic studies, given the rigorous approach we took to directly measuring biological endpoints, linking molecular and morphologic biomarkers, and measuring exposures and outcomes within a critical developmental window.

Our additional analysis of matched maternal-fetal tissue samples revealed reasonably consistent patterns of association between molecular placental biomarkers and PBDE measurements across maternal and fetal tissues, with stronger PBDE associations observed in fetal liver

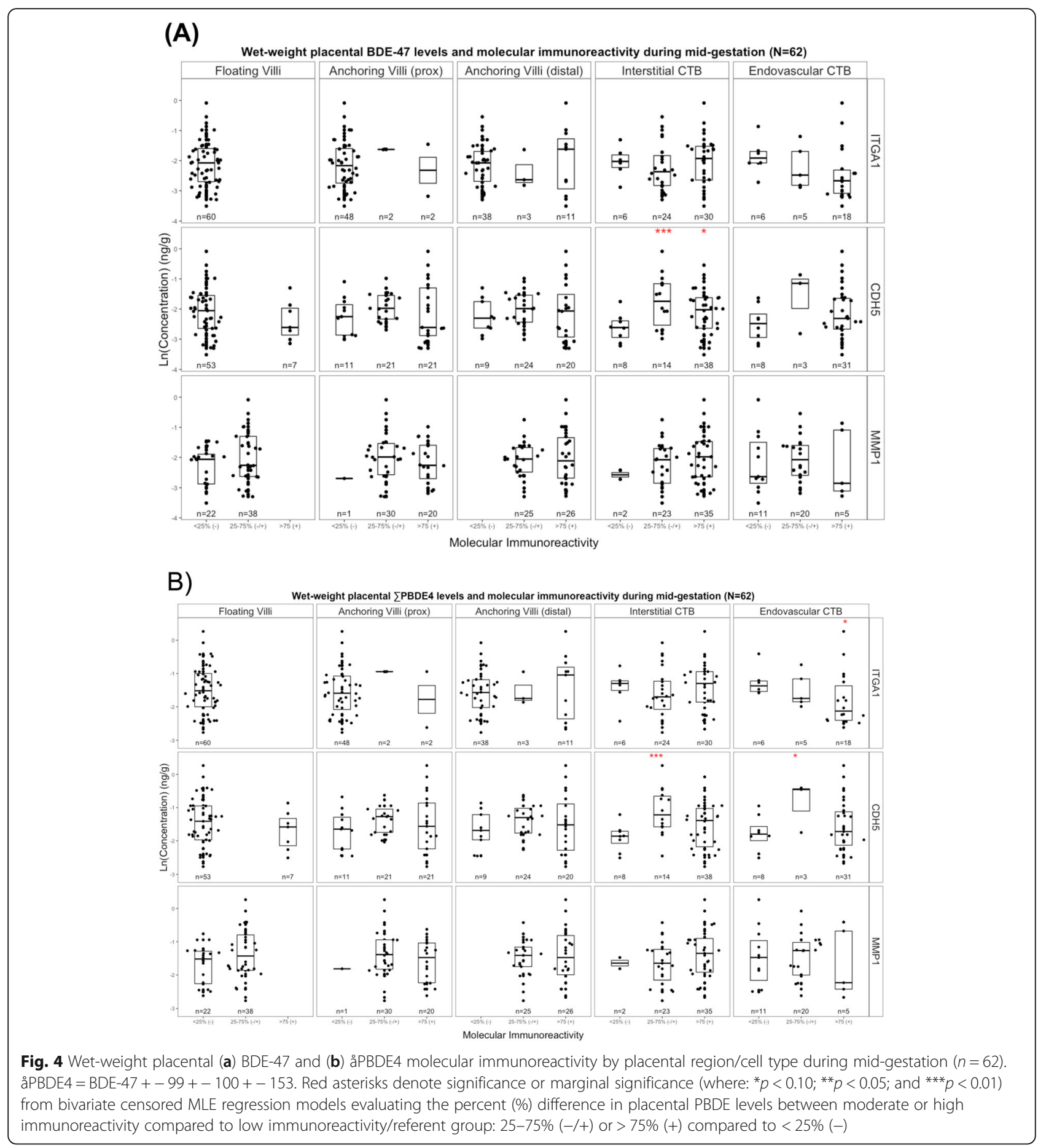


samples. This may not be surprising, given the extreme interdependence of placental and fetal development during pregnancy, as well as the intricate coordination of hormones and molecular signaling pathways that drive biological changes and physiological adaptations between maternal, placental, and fetal compartments. Further efforts to identify possible mechanisms of placental PBDE toxicity relevant to fetal exposures are warranted.

Although we found no association between placental PBDE concentrations and the morphological endpoints we analyzed, our study is one of the first to characterize several important features of placental morphology during mid-gestation, a critical time of placentation about which current biological data is limited. Additionally, null associations are consistent with the fact that the morphological changes we examined are indicative of severe placental disease and damage, which may not be apparent until later in pregnancy. Given that typical PBDE exposures during pregnancy are not consistently associated with overt pregnancy complications, it is likely that these (and other environmental chemicals) have more subtle effects on placental tissues that are difficult to discern with the morphological features we selected. Identifying the most sensitive upstream physiological endpoints during mid-gestation that are relevant for placenta-mediated diseases would improve the utility of examining morphological features in future studies. Further integrating these physiological parameters with additional molecular and clinical measures that have been shown to improve diagnostic accuracy for complications like preeclampsia and fetal growth restriction (e.g., placental growth factor, fms-like tyrosine kinase-1, and advanced ultrasound techniques) [19] would also strengthen future research in this area. Moreover, efforts to identify circulating measures or imaging biomarkers that are more practical to measure in a larger sample would further advance the impact of studies conducted on placental tissues (such as ours) which are not accessible in intact pregnancies. Nevertheless, the use of primary human placental tissues is a strength of this study, as it enables direct correlation of the relationships examined and reduces uncertainties in extrapolation. As a complement to these studies, experimental investigations using primary cells, cell lines or animal models can be utilized to investigate relationships between exposure and placental dysfunction while controlling for many of the inherent challenges of observational studies (e.g., background exposures, gestational age and genetic variation, obtaining tissue).

In the time since we initiated this analysis, the application of new technologies for global profiling have emerged as a powerful way of answering important questions that are being raised about the effects of environmental chemical exposures on placental development.
Many of these technologies could be applied to studies of similar design to ours in the future. For example, laser capture of specific trophoblast populations combined with global protein profiling by mass spectrometry could reveal interesting associations with environmental chemical exposures that are not biased toward molecules with well-studied roles in CTB differentiation and placental development. Also, discovering additional biomarkers of placental pathways (e.g., oxidative stress, inflammation, angiogenesis, and/or hormone signaling) that are perturbed by PBDE exposures could yield valuable insights into how these and other environmental chemicals impact pregnancy. Finally, given the explosive rate of placental development, it will be interesting to apply this approach across gestation to identify particularly important periods of vulnerability.

\section{Conclusions}

We conducted a novel epidemiologic study using a rigorous approach for measuring biomarkers of PBDE exposure and placental development and disease with high resolution and precision. We examined multiple molecular indicators of placenta-mediated pregnancy complications that may also be sensitive indicators of maternal-fetal PBDE exposures. This novel approach can be applied more widely to examine additional molecular biomarkers of placental development and function (e.g., placental hormones, angiogenic factors, etc.) and/or other early indicators of pregnancy complications like preeclampsia (e.g., blood pressure) in epidemiologic studies of chemical exposures and adverse maternal-fetal health outcomes. Our findings indicate that placental biomarkers of development and disease could be useful barometers of exposure to PBDEs, a paradigm that could be extended to other environmental chemicals and placental stage-specific antigens.

\section{Supplementary information}

Supplementary information accompanies this paper at https://doi.org/10. 1186/s12940-020-00617-7.

\footnotetext{
Additional file 1: Table S1. Molecular and morphological biomarkers of placental disease and development during mid-gestation. Table S2. Primary antibodies used for immunolocalization assessments. Table S3. Molecular immunoreactivity and low/high PBDE exposure by placental region/cell type during mid-gestation $(n=62)$. Table S4. Detection frequencies for 19 PBDE congeners in matched samples of maternal serum, placenta, and fetal liver during mid-gestation $(n=130)$. Table S5. Correlation (unadjusted $p$-value) ${ }^{a}$ of molecular and morphological biomarkers during mid-gestation $(n=62)$. Table S6. Correlation (FDR estimate) ${ }^{a}$ of molecular and morphological placental biomarkers during mid-gestation $(n=62)$. Table S7. Correlation (unadjusted $p$-values and FDR) ${ }^{a}$ of placental biomarkers and wet-weight PBDE levels during mid-gestation $(n=62)$.

Additional file 2: Figure S1. Diagram of study framework illustrating approach to evaluating relationships between biomarkers of chemical exposure and potential biomarkers of placental development and disease. Figure S2. Immunoreactivity of ITGA1 at the maternal-fetal
} 
interface. Representative images of floating (A-C) and anchoring (D-F) villi, interstitial invading CTBs (CTBi) of the decidua (G-I), and endovascular CTBs (CTBe) lining a uterine artery (J-L). Tissue sections of 2 nd trimester samples were immunostained for ITGA1 (green) and co-stained with anti-CK (trophoblast marker, red) and DAPI (nuclear dye, blue). Images represent typical profiles of ITGA1 expression. Bars $=100 \mu \mathrm{m}$. Figure S3. Immunoreactivity of $\mathrm{CDH} 5$ at the maternal-fetal interface. Representative images of floating $(\mathbf{A}-\mathbf{C})$ and anchoring (D-F) villi, interstitial invading CTBs (CTBi) of the decidua (G-I), and endovascular CTBs (CTBe) lining a uterine artery (J-L). Tissue sections of 2 nd trimester samples were immunostained for ITGA1 (green) and co-stained with anti-CK (trophoblast marker, red) and DAPI (nuclear dye, blue). Images represent typical profile of $\mathrm{CDH} 5$ expression. Bars $=100 \mu \mathrm{m}$. Figure S4. Immunoreactivity of MMP1 at the maternal-fetal interface. Representative images of floating (A-C) and anchoring (D-F) villi, interstitial invading CTBs (CTBi) of the decidua (G-I), and endovascular CTBs (CTBe) lining a uterine artery (J-L). Tissue sections of 2 nd trimester samples were immunostained for ITGA1 (green) and co-stained with anti-CK (trophoblast marker, red) and DAPI (nuclear dye, blue). Images represent typical profile of MMP1 expression. Bars $=100 \mu \mathrm{m}$. Figure S5. Representative morphological features of the maternalfetal interface. Representative images of morphological features that were scored in human placental biopsies: (A) perivillous fibrinoid deposition surrounding floating villi, (B) fibrinoid deposition, (C) leukocyte (white blood cell) infiltration of the basal plate, and (D) CTB remodeling of uterine arteries. Black arrows and dotted black lines indicate the feature of interest.

Additional file 3.

\section{Abbreviations}

AV: Anchoring villi; BP: Basal plate; BMI: Body mass index; $C D H 5$ : Vascular endothelial-cadherin; CK: Cytokeratin; CTB: Cytotrophoblast; dAV: Distal anchoring villi; ECM: Extracellular matrix; eCTB: Endovascular CTB; EDC: Endocrine disrupting chemical; FV: Floating villi; H\&E: Hematoxylin and Eosin; iCTB: Invasive/extravillous CTB; IF: Immunofluorescence; ITGA1: Integrin alpha-1; MDL: Method detection limit; MLE: Maximum likelihood estimation; MMP1: Metalloproteinase-1; MTBE: Methyl tert-butyl ether; pAV: Proximal anchoring villi; PBDE: Polybrominated diphenyl ethers; PFAS: Per- and polyfluorinated alkyl substances; SES: Socioeconomic status; STB: Syncytiotrophoblast; TH: Thyroid hormone; UCSF: University of California, San Francisco; US: United States; WBC: White blood cell; WOC: Women's Options Center

\section{Acknowledgements}

We thank all research participants and clinical staff at San Francisco General Hospital Women's Options Center (WOC) for assistance and support in this study. We specifically thank Erin DeMicco for study coordination and Cheryl Godwin de Medina for study recruitment and sample collection in the WOC.

\section{Authors' contributions}

JRV, JFR, SJF, and TJW designed and directed the project; YZ, JFR, KAP, EK, SB, and RA performed histological and molecular assessments of placental samples; JSP supervised and SCS and JF performed the chemical analysis of biological samples; JRV, SS, and JFR conducted the statistical analyses; JRV, JFR, SS, SLG, SJF, and TJW interpreted the experimental results; JRV and JFR constructed the figures; JRV, JFR, SJF, and TJW wrote the article. All authors read and approved the final manuscript.

\section{Funding}

This work was supported by the U.S. Environmental Protection Agency (No. RD-83543301) and the National Institute of Environmental Health Sciences (P01ES022841, R00ES023846), and the National Institute of Allergy and Infectious Diseases (K08Al141728 to SLG). These funding sources had no involvement in the conduct of the research or preparation of this article.

\section{Availability of data and materials}

The datasets used and/or analyzed during the current study are available from the corresponding author on reasonable request.

\section{Ethics approval and consent to participate}

This study was approved by the University of California, San Francisco Institutional Review Board (UCSF IRB). Written and verbal consent were obtained from study participants prior to sample collection in accordance with the Declaration of Helsinki.

\section{Consent for publication}

Not applicable.

\section{Competing interests}

The authors declare they have no actual or potential competing financial interests.

\section{Author details}

'Program on Reproductive Health and the Environment, UCSF Department of Obstetrics, Gynecology \& Reproductive Sciences, University of California, Mailstop 0132, 550 16th Street, 7th Floor, San Francisco, CA 94143, USA. ${ }^{2}$ Center for Reproductive Sciences and Department of Obstetrics, Gynecology \& Reproductive Sciences, University of California, 513 Parnassus Avenue, San Francisco, CA 94143, USA. ${ }^{3}$ Division of Maternal-Fetal Medicine and Department of Obstetrics, Gynecology \& Reproductive Sciences, University of California, 550 16th Street, 7th Floor, San Francisco, CA 94143, USA. ${ }^{4}$ Department of Preventive Medicine, University of Tennessee Health Science Center, 66 North Pauline St, Memphis, TN 38163, USA. ${ }^{5}$ California Environmental Protection Agency, Department of Toxic Substances Control, Environmental Chemistry Laboratory, 700 Heinz Ave \# 200, Berkeley, CA 94710, USA.

Received: 10 December 2019 Accepted: 21 May 2020 Published online: 03 June 2020

\section{References}

1. Leddy MA, Power ML, Schulkin J. The impact of maternal obesity on maternal and fetal health. Rev Obstet Gynecol. 2008;1:170-8.

2. ACOG. Committee opinion no. 575: exposure to toxic environmental agents. American College of Obstetricians and Gynecologists (ACOG). Obstet Gynecol. 2013:122:931-4.

3. Ferguson KK, Chin HB. Environmental chemicals and preterm birth: biological mechanisms and the state of the science. Curr Epidemiol Rep. 2017:4:56-71.

4. Varshavsky J. Heightened susceptibility: a review of how pregnancy and chemical exposures influence maternal health. Reprod Toxicol. 2019;508906238(18):30434-9.

5. Napso T, Yong HEJ, Lopez-Tello J, Sferruzzi-Perri AN. The role of placental hormones in mediating maternal adaptations to support pregnancy and lactation. Front Physiol. 2018:9 [cited 2018 Sep 5] Available from: https:// www.frontiersin.org/articles/10.3389/fphys.2018.01091/full.

6. Talbot L, Maclennan K. Physiology of pregnancy. Anaesthesia Intensive Care Med. 2016;17:341-5

7. Zhou Y, Fisher SJ, Janatpour M, Genbacev O, Dejana E, Wheelock M, et al. Human cytotrophoblasts adopt a vascular phenotype as they differentiate. A strategy for successful endovascular invasion? J Clin Invest. 1997;99:213951.

8. Fisher SJ. Why is placentation abnormal in preeclampsia? Am J Obstet Gynecol. 2015;213:S115-22.

9. Damsky CH, Librach C, Lim KH, Fitzgerald ML, McMaster MT, Janatpour M, et al. Integrin switching regulates normal trophoblast invasion. Development. 1994;120:3657-66.

10. Zhou Y, Damsky CH, Chiu K, Roberts JM, Fisher SJ. Preeclampsia is associated with abnormal expression of adhesion molecules by invasive cytotrophoblasts. J Clin Invest. 1993;91:950-60.

11. Zhou Y, Damsky CH, Fisher SJ. Preeclampsia is associated with failure of human cytotrophoblasts to mimic a vascular adhesion phenotype. One cause of defective endovascular invasion in this syndrome? J Clin Invest. 1997;99:2152-64.

12. Kaufmann P, Black S, Huppertz B. Endovascular trophoblast invasion: implications for the pathogenesis of intrauterine growth retardation and preeclampsia. Biol Reprod. 2003;69:1-7.

13. Blackwell S, Romero R, Chaiworapongsa T, Kim YM, Bujold E, Espinoza J, et al. Maternal and fetal inflammatory responses in unexplained fetal death. J Matern Fetal Neonatal Med. 2003;14:151-7. 
14. Romero R, Kusanovic JP, Chaiworapongsa T, Hassan SS. Placental bed disorders in preterm labor, preterm PROM, spontaneous abortion and abruptio placentae. Best Pract Res Clin Obstet Gynaecol. 2011;25:313-27.

15. Garrido-Gomez T, Ona K, Kapidzic M, Gormley M, Simón C, Genbacev O, et al. Severe pre-eclampsia is associated with alterations in cytotrophoblasts of the smooth chorion. Development. 2017;144:767-77.

16. Kim YM, Bujold E, Chaiworapongsa T, Gomez R, Yoon BH, Thaler HT, et al. Failure of physiologic transformation of the spiral arteries in patients with preterm labor and intact membranes. Am J Obstet Gynecol. 2003;189:1063-9.

17. Liu S, Diao L, Huang C, Li Y, Zeng Y, Kwak-Kim JYH. The role of decidual immune cells on human pregnancy. J Reprod Immunol. 2017;124:44-53.

18. Kim CJ, Romero R, Chaemsaithong P, Kim J-S. Chronic inflammation of the placenta: definition, classification, pathogenesis, and clinical significance. Am J Obstet Gynecol. 2015;213:S53-69.

19. Kingdom JC, Audette MC, Hobson SR, Windrim RC, Morgen E. A placenta clinic approach to the diagnosis and management of fetal growth restriction. Am J Obstet Gynecol. 2018;218:S803-17.

20. Phuapittayalert L, Norkaew T, Supanpaiboon W, Chuenchoojit S, Hipkeao W, Swaddiwudhipong W, et al. Increasing of syncytial knot and Fibrinoid deposit in high-cd accumulated human placentas. Int J Morphol. 2013;31: 1210-5.

21. Poropat AE, Laidlaw MAS, Lanphear B, Ball A, Mielke HW. Blood lead and preeclampsia: a meta-analysis and review of implications. Environ Res. 2018; 160:12-9.

22. Ilekis JV, Tsilou E, Fisher S, Abrahams VM, Soares MJ, Cross JC, et al. Placental origins of adverse pregnancy outcomes: potential molecular targets: an executive workshop summary of the Eunice Kennedy Shriver National Institute of Child Health and Human Development. Am J Obstet Gynecol. 2016;215:S1-46.

23. ATSDR. Public Health Statement: Polybrominated Diphenyl Ethers (PBDEs) [Internet]. Department of Health and Human Services, Public Health Service, Agency for Toxic Substances and Disease Registry, Division of Toxicology and Human Health Sciences; 2017. Available from: https://www.atsdr.cdc. gov/PHS/PHS.asp?id=1449\&tid=183.

24. Leonetti C, Butt CM, Hoffman K, Miranda ML, Stapleton HM. Concentrations of polybrominated diphenyl ethers (PBDEs) and 2,4,6-tribromophenol in human placental tissues. Environ Int. 2016;88:23-9.

25. Zota AR, Mitro SD, Robinson JF, Hamilton EG, Park J-S, Parry E, et al. Polybrominated diphenyl ethers (PBDEs) and hydroxylated PBDE metabolites (OH-PBDEs) in maternal and fetal tissues, and associations with fetal cytochrome P450 gene expression. Environ Int. 2018;112:269-78.

26. Zhao X, Peng S, Xiang Y, Yang Y, Li J, Shan Z, et al. Correlation between prenatal exposure to Polybrominated Diphenyl ethers (PBDEs) and infant birth outcomes: a meta-analysis and an experimental study. Int J Environ Res Public Health. 2017;14:268

27. Lam J, Lanphear BP, Bellinger D, Axelrad DA, McPartland J, Sutton P, et al. Developmental PBDE exposure and IQ/ADHD in childhood: a systematic review and meta-analysis. Environ Health Perspect. 2017:125 [cited 2019 Mar 21]. Available from: https://www.ncbi.nlm.nih.gov/pmc/articles/PMC5783655/.

28. Zota AR, Rudel RA, Morello-Frosch RA, Brody JG. Elevated house dust and serum concentrations of PBDEs in California: unintended consequences of furniture flammability standards? Environ Sci Technol. 2008;42:8158-64.

29. Eslami B, Malekafzali H, Rastkari N, Rashidi BH, Djazayeri A, Naddafi K Association of serum concentrations of persistent organic pollutants (POPs) and risk of pre-eclampsia: a case-control study. J Environ Health Sci Eng. 2016;14:17.

30. Smarr MM, Grantz KL, Zhang C, Sundaram R, Maisog JM, Barr DB, et al. Persistent organic pollutants and pregnancy complications. Sci Total Environ. 2016:551-552:285-91.

31. Yu L, Han Z, Liu C. A review on the effects of PBDEs on thyroid and reproduction systems in fish. Gen Comp Endocrinol. 2015;219:64-73.

32. Park H-R, Loch-Caruso R. Protective effect of ( \pm )a-tocopherol on brominated diphenyl ether-47-stimulated prostaglandin pathways in human extravillous trophoblasts in vitro. Toxicol in Vitro. 2015;29:1309-18.

33. Park H-R, Kamau PW, Korte C, Loch-Caruso R. Tetrabromobisphenol a activates inflammatory pathways in human first trimester Extravillous Trophoblasts in vitro. Reprod Toxicol. 2014;50:154-62.

34. Park H-R, Loch-Caruso R. Protective effect of nuclear factor E2-related factor 2 on inflammatory cytokine response to brominated Diphenyl Ether-47 in the HTR-8/SVneo human first trimester Extravillous Trophoblast cell line. Toxicol Appl Pharmacol. 2014;281:67-77.
35. Peltier MR, Klimova NG, Arita Y, Gurzenda EM, Murthy A, Chawala K, et al. Polybrominated diphenyl ethers enhance the production of proinflammatory cytokines by the placenta. Placenta. 2012;33:745-9.

36. Arita Y, Yeh C, Thoma T, Getahun D, Menon R, Peltier MR. Effect of polybrominated diphenyl ether congeners on placental cytokine production. J Reprod Immunol. 2018;125:72-9.

37. Leonetti CP, Butt CM, Stapleton HM. Disruption of thyroid hormone sulfotransferase activity by brominated flame retardant chemicals in the human choriocarcinoma placenta cell line,BeWo. Chemosphere. 2018;197: $81-8$.

38. Zhu Y, Tan YQ, Leung LK. Exposure to 2,2',4,4'-tetrabromodiphenyl ether at late gestation modulates placental signaling molecules in the mouse model. Chemosphere. 2017;181:289-95.

39. Zhu $Y$, Tan $Y Q$, Wang CC, Leung LK. The flame retardant $2,2^{\prime}, 4,4^{\prime}-$ Tetrabromodiphenyl ether enhances the expression of corticotropinreleasing hormone in the placental cell model JEG-3. Chemosphere. 2017; 174:499-505.

40. Robinson JF, Kapidzic M, Hamilton EG, Chen H, Puckett KW, Zhou Y, et al. Genomic profiling of BDE-47 effects on human placental Cytotrophoblasts. Toxicol Sci. 2019;167:211-26.

41. Park H-R, Kamau PW, Loch-Caruso R. Involvement of reactive oxygen species in brominated diphenyl ether-47-induced inflammatory cytokine release from human extravillous trophoblasts in vitro. Toxicol Appl Pharmacol. 2014;274:283-92.

42. Damsky CH, Fitzgerald ML, Fisher SJ. Distribution patterns of extracellular matrix components and adhesion receptors are intricately modulated during first trimester cytotrophoblast differentiation along the invasive pathway, in vivo. J Clin Invest. 1992;89:210-22.

43. Morello-Frosch R, Cushing LJ, Jesdale BM, Schwartz JM, Guo W, Guo T, et al. Environmental Chemicals in an Urban Population of pregnant women and their newborns from San Francisco. Environ Sci Technol. 2016;50: 12464-72.

44. Whitehead TP, Smith SC, Park J-S, Petreas MX, Rappaport SM, Metayer C. Concentrations of persistent organic pollutants in California women's serum and residential dust. Environ Res. 2015;136:57-66.

45. Phillips DL, Smith AB, Burse WW, Steele GK, Needham LL, Hannon WH. Halflife of polychlorinated biphenyls in occupationally exposed workers. Arch Environ Health. 1989:44:351-4.

46. Vettraino IM, Roby J, Tolley T, Parks WC. Collagenase-I, stromelysin-I, and matrilysin are expressed within the placenta during multiple stages of human pregnancy. Placenta. 1996;17:557-63.

47. Zhou Y, Bellingard V, Feng K-T, McMaster M, Fisher SJ. Human cytotrophoblasts promote endothelial survival and vascular remodeling through secretion of Ang2, PIGF, and VEGF-C. Dev Biol. 2003;263:114-25.

48. Schisterman EF, Whitcomb BW, Buck Louis GM, Louis TA. Lipid adjustment in the analysis of environmental contaminants and human health risks. Environ Health Perspect. 2005;113:853-7.

49. O'Brien KM, Upson K, Cook NR, Weinberg CR. Environmental Chemicals in Urine and Blood: improving methods for Creatinine and lipid adjustment. Environ Health Perspect. 2016;124:220-7.

50. Helsel DR. Nondetects and data analysis: statistics for environmental data. Hoboken, New Jersey: Wiley; 2005.

51. R Core Team. R: A language and environment for statistical computing. Vienna, Austria; 2018. Available from: https://www.R-project.org/.

52. Lopaka Lee. NADA: nondetects and data analysis for environmental data. 2017. Available from: https://CRAN.R-project.org/package=NADA.

53. Akritas MG, Murphy SA, LaValley MP. The Theil-Sen estimator with doubly censored data and applications to astronomy. J Am Stat Assoc 1995;90:170177.

54. Benjamini $Y$, Hochberg Y. Controlling the false discovery rate: a practical and powerful approach to multiple testing. J Royal Stat Soc. 1995;57:289_ 300.

55. Varshavsky J, Sen S, Robinson JF, Smith SC, Frankenfield J, Wang Y, et al. Racial/Ethnic and Geographic Differences in Polybrominated Diphenyl Ether (PBDE) Levels across Maternal, Placental, and Fetal Tissues during Midgestation. Scientific Reports Accepted, In press. 2020.

56. Zhu J-Y, Pang Z-J, Yu Y. Regulation of Trophoblast invasion: the role of matrix Metalloproteinases. Rev Obstet Gynecol. 2012;5:e137-43.

57. Bulla R, Villa A, Bossi F, Cassetti A, Radillo O, Spessotto P, et al. VE-cadherin is a critical molecule for trophoblast-endothelial cell interaction in decidual spiral arteries. Exp Cell Res. 2005;303:101-13. 
58. Lan X, Fu L-J, Zhang J, Liu X-Q, Zhang H-J, Zhang X, et al. Bisphenol a exposure promotes HTR-8/SVneo cell migration and impairs mouse placentation involving upregulation of integrin- $\beta 1$ and MMP-9 and stimulation of MAPK and PI3K signaling pathways. Oncotarget. 2017;8: 51507-21.

59. Haider S, Pal R. Integrated analysis of Transcriptomic and proteomic data. Curr Genomics. 2013;14:91-110.

\section{Publisher's Note}

Springer Nature remains neutral with regard to jurisdictional claims in published maps and institutional affiliations.

Ready to submit your research? Choose BMC and benefit from:

- fast, convenient online submission

- thorough peer review by experienced researchers in your field

- rapid publication on acceptance

- support for research data, including large and complex data types

- gold Open Access which fosters wider collaboration and increased citations

- maximum visibility for your research: over $100 \mathrm{M}$ website views per year

At $\mathrm{BMC}$, research is always in progress.

Learn more biomedcentral.com/submissions 